\title{
Equilíbrio, mobilidade funcional e qualidade de vida em idosos participantes e não participantes de um centro de convivência
}

\author{
Balance, functional mobility and quality of life in eldery participants and \\ non-participants of a community center
}

\author{
Maiara Lohn de Farias ${ }^{1}$, Lisiane Piazza Luza² $₫$, Bianca Andrade de Sousa ${ }^{3}$, Ediane Roberge Zampirolo ${ }^{1}$ \\ ${ }^{1}$ Departamento de Fisioterapia do Instituto de Ensino Superior da Grande Florianópolis (IESGF). Florianópolis, SC. \\ ${ }^{2}$ Departamento de Fisioterapia da Universidade do Estado de Santa Catarina (UDESC). Florianópolis, SC. \\ ${ }^{3}$ Departamento de Educação Física da UDESC. Florianópolis, SC.
}

\section{RESUMO}

OBJETIVOS: Avaliar o equilíbrio, a mobilidade funcional e a qualidade de vida em idosos participantes e não participantes de um centro de convivência.

MÉTODOS: Foram avaliados sujeitos com idade igual ou acima de 60 anos, participantes de um centro de convivência para idosos da cidade de Santo Amaro da Imperatriz, em Santa Catarina (Grupo de Participantes: GP). Como grupo controle, para comparação, foram incluídos idosos moradores da mesma comunidade, os quais não participavam de nenhum centro de convivência (Grupo de Não Participantes: GNP). Foram excluídos sujeitos com incapacidade de locomoção, doenças neurológicas que afetassem a marcha ou o equilíbrio e incapacidade para compreender os comandos gerais indispensáveis aos testes. A amostra foi não probabilística intencional. Para avaliação do equilíbrio foi aplicada a Escala de Equilíbrio de Berg e para mobilidade funcional os testes Timed Up and Go e de Alcance Funcional Anterior. A qualidade de vida foi avaliada pelo questionário SF-36. Os dados foram tratados pela estatística descritiva e inferencial, sendo considerados significativos valores com $\mathrm{p} \leq 0,05$.

RESULTADOS: Participaram 56 idosos, sendo 28 do GP e 28 do GNP. Por meio da Escala de Equilíbrio de Berg verificou-se melhor equilíbrio no GP (média 53,2 $\pm 2,1$ pontos) em comparação ao GNP (média 48,8 $\pm 6,2$ pontos) ( $\mathrm{p}=0,001$ ). No teste Timed Up and Go, o GP levou em média menos tempo para realizar o teste $(9,5 \pm 1,5$ segundos $)$ do que o $\operatorname{GNP}(13,1 \pm 5,1$ segundos $)(p=0,001)$. Observou-se melhor qualidade de vida em todos os domínios do SF-36 no GP em relação ao GNP.

CONCLUSÕES: Idosos que participavam de centro de convivência apresentaram melhor equilíbrio, mobilidade funcional e qualidade de vida do que idosos da mesma comunidade não participantes de centros de convivência.

DESCRITORES: idoso; atividade motora; centros comunitários para idosos; equilíbrio postural; qualidade de vida.

\section{ABSTRACT}

AIMS: To evaluate balance, functional mobility and quality of life in elderly participants and non-participants of a senior citizen center. METHODS: Subjects aged 60 or over, participating in a senior citizen center in the city of Santo Amaro da Imperatriz, in Santa Catarina (Participating Group: PG) were evaluated. As a control group for comparison, elderly residents of the same community who did not participate in any senior center (Non-Participating Group: NPG) were included. Subjects with locomotion disabilities, neurological diseases that affected the gait or balance, and inability to understand the general commands indispensable to the tests were excluded. The sample was non-probabilistic intentional. To evaluate the balance, the Berg Balance Scale was applied, and for assess functional mobility we used the Timed Up and Go and the Anterior Functional Scope tests. Quality of life was assessed by the SF-36 questionnaire. The data were treated by descriptive and inferential statistics, considering $\mathrm{p} \leq 0.05$ as significant.

RESULTS: Fifty-six elderly participated, being 28 of PG and 28 of NPG. By means of the Berg Balance Scale, we found a better balance in PG (mean 53.2 \pm 2.1 points) compared to NPG (mean $48.8 \pm 6.2$ points) $(\mathrm{p}=0.001)$. In the Timed Up and Go test, PG spent in average less time to perform the test $(9.5 \pm 1.5$ seconds) than NPG (13.1 \pm 5.1 seconds) $(\mathrm{p}=0.001)$. We observed a better quality of life in all domains of SF-36 in PG when compared to NPG $(\mathrm{p}<0.05)$.

CONCLUSIONS: Elderly people who participated in a senior citizen center presented better balance, functional mobility and quality of life than elderly people from the same community who did not participate in senior centers.

KEY WORDS: aged; motor activity; senior centers; postural balance; quality of life. 
Abreviaturas: QV, qualidade de vida; GP, grupo de participantes do centro de convivência para idosos; GNP, grupo de não participantes de um centro de convivência, constituído por idosos moradores da comunidade; EEB, escala de equilíbrio de Berg; TUG, Teste Time Up and Go; TAF, teste de alcance funcional anterior; SF-36, 36-Item Short Form Health Survey.

\section{INTRODUÇÃO}

O crescimento da população idosa é uma realidade demográfica cada vez mais significativa, que vem ocorrendo devido à redução na taxa de fecundidade, aumento da expectativa de vida e redução da mortalidade [1]. No Brasil, o número de idosos atingia três milhões de pessoas em 1960, sete milhões em 1975, dez milhões e meio em 1991, quatorze milhões em 2002 e calcula-se que poderá atingir a faixa dos trinta e dois milhões em 2020 [2].

O processo do envelhecimento pode ocasionar inúmeras modificações morfológicas, bioquímicas, psicológicas e funcionais no organismo do indivíduo [3]. Dentre tais comprometimentos, destacase a instabilidade postural, que ocorre devido às alterações dos sistemas sensorial e motor, levando a uma redução do equilíbrio [4]. O comprometimento do equilíbrio corporal pode interferir na mobilidade funcional, gerando também maior predisposição a quedas, fraturas e receio de cair novamente [5-8]. Esses fatores podem conduzir também à redução na qualidade de vida (QV) dos idosos, uma vez que esta é determinada, em grande parte, pela capacidade do idoso em manter sua autonomia e independência. A maioria das pessoas teme a velhice pelas possibilidades de se tornarem dependentes por determinada doença ou não poderem realizar suas atividades cotidianas por redução da capacidade funcional $[9,10]$.

Nesse contexto, os grupos de convivência de idosos vão de encontro à promoção do envelhecimento ativo, com objetivo de preservação das capacidades e do potencial de desenvolvimento do indivíduo idoso. Esses grupos desempenham atividades múltiplas, de cunho recreativo, cultural, social, educativo e de promoção da saúde [11].

O objetivo deste estudo foi comparar o equilíbrio, a mobilidade funcional e a QV em idosos participantes e não participantes de um centro de convivência.

\section{MÉTODOS}

Foi realizado um estudo transversal, aprovado pelo Comitê de Ética em Pesquisa da Universidade Paulista sob o parecer $n^{\circ}$ 1.584.312. Todos os sujeitos foram informados sobre os objetivos e procedimentos da pesquisa, sendo garantido a todos que não seriam expostos a riscos de nenhuma natureza, preservando sua integridade física, mental e emocional, e solicitando a assinatura do Termo de Consentimento Livre e Esclarecido (TCLE).

$\mathrm{O}$ estudo foi realizado na cidade de Santo Amaro da Imperatriz, estado de Santa Catarina. O período de coleta dos dados foi entre julho e agosto de 2016. Os participantes foram selecionados de forma não probabilística intencional. Os critérios de inclusão foram idade igual ou acima de 60 anos e capacidade para assinar o termo de consentimento. Não foram incluídos idosos com incapacidade de locomoção, presença de doenças neurológicas que afetassem a marcha e o equilíbrio e incapacidade para compreender os comandos gerais indispensáveis para realização dos testes.

Para o grupo de estudo foram selecionados participantes do Centro de Convivência da Melhor Idade, da cidade de Santo Amaro da Imperatriz, que reúne semanalmente idosos com o intuito de promover atividades físicas, como caminhadas, alongamentos de grandes grupos musculares, mobilizações articulares ativas e fortalecimento muscular através de exercícios resistidos ou com o peso do próprio membro. Possibilita também a participação e organização de eventos na cidade. Inicialmente entrou-se em contato com a responsável pelo Centro de Convivência da Melhor Idade, solicitando o aval para a execução do estudo. Após a autorização da responsável, foram selecionados os idosos que se enquadraram nos critérios de inclusão da pesquisa e estes foram convidados a participar. Esse grupo foi denominado de Grupo de Participantes (GP).

Para o Grupo de Não Participantes (GNP) foram selecionados idosos da comunidade que não participavam de nenhum grupo de convivência. A divulgação foi feita por meio de folders informativos, distribuídos entre os moradores da cidade e também nas redes sociais, convidando os idosos a realizarem as avaliações. Àqueles que demonstraram interesse por meio de contato telefônico com os pesquisadores, foi agendada uma entrevista na residência do idoso, para explicar a pesquisa e agendar as avaliações com os que concordaram.

Para caracterização dos sujeitos foi aplicada uma ficha de avaliação elaborada previamente pelos pesquisadores, com as seguintes informações: idade, gênero, se realizava ou não fisioterapia, se praticava ou não atividade física, histórico de quedas, medicações e doenças associadas, sendo todas questões abertas. $\mathrm{Na}$ execução do estudo foram utilizados os seguintes 
materiais: cronômetro, fita métrica, um degrau e uma cadeira. Para avaliação do equilíbrio foi utilizada a Escala de Equilíbrio de Berg (EEB) [12], considerada um instrumento confiável e que permite determinar os fatores de risco para perda da independência e para quedas em idosos. Esse instrumento possui 14 itens, cada um valendo de 0 a 4 pontos, permitindo chegar ao escore total de 56 pontos, que representa nenhuma alteração de equilíbrio. As 14 tarefas que compõem a EEB são as seguintes: levantar-se de uma cadeira, permanecer em pé sem apoio, sentar-se na cadeira, transferir-se de uma cadeira para a outra, permanecer em pé com os olhos fechados, permanecer em pé com os pés juntos, alcançar a frente permanecendo em pé, pegar um objeto no chão, virar-se e olhar para trás, girar 360 graus, posicionar os pés alternadamente em um degrau, permanecer em pé com um pé na frente do outro, permanecer em pé sobre uma perna [12].

Para mobilidade funcional foram utilizados o teste Timed Up and Go (TUG) criado por Podsiadlo e Richardson [13], e o Teste de Alcance Funcional (TAF), proposto por Duncan [14]. Para realização do TUG, o idoso é orientado a levantar-se de uma cadeira com encosto, caminhar três metros, dar a volta, retornar a cadeira e sentar com as costas apoiadas no encosto, sendo o tempo para realização da tarefa cronometrado. A realização do teste em até 10 segundos é considerada normal para indivíduos independentes e sem risco de quedas, entre 11 e 20 segundos é o esperado para idosos frágeis, com independência parcial e com baixo risco de quedas, e acima de 20 segundos indica déficit importante da mobilidade física e risco de quedas [13].

O TAF tem por objetivo determinar o quanto o idoso é capaz de se deslocar dentro do limite de estabilidade anterior, sendo muito utilizado para verificar o risco de quedas em idosos. Coloca-se uma fita métrica em uma parede, sendo a fita paralela ao chão e na altura do acrômio de cada sujeito avaliado. É solicitado que o sujeito fique descalço, com os pés paralelos, perpendicular à parede e próximo do início da fita métrica. É então solicitado que realize uma inclinação anterior, com os cotovelos estendidos e ombros a 90 graus, paralelos à fita métrica, porém sem tocá-la, e o pesquisador verifica o deslocamento anterior do sujeito sobre a fita métrica. A diferença entre a medida inicial e final registrada na fita métrica representa o deslocamento, que quando menor que 15 centímetros indica fragilidade do indivíduo e risco de quedas [14]. No presente estudo, o teste foi realizado três vezes e seu resultado foi obtido pela média das três tentativas.
Para avaliar a QV foi utilizado o Questionário SF-36 (36-Item Short Form Health Survey), o qual foi traduzido e validado no Brasil por Ciconelli et al. (1999) [15], sendo este um instrumento genérico de avaliação da QV, formado por 36 itens, os quais estão englobados em oito domínios: capacidade funcional, aspectos físicos, dor, estado geral de saúde, vitalidade, aspectos sociais, emocionais e saúde mental. O escore é pontuado para cada domínio, podendo variar de zero a 100 , sendo que zero representa o pior escore e 100 o melhor [16].

No dia das avaliações, após a assinatura do TCLE, foram aplicados, além da ficha de informações, o questionário SF-36, a EEB e os testes TUG e TAF, sendo estes realizados em ordem randomizada, por sorteio. As avaliações foram feitas nos períodos matutino e vespertino, com temperatura ambiente variando entre $20-25^{\circ} \mathrm{C}$ e todos os sujeitos utilizavam roupas confortáveis a fim de possibilitar a realização dos testes. Antes do início das coletas foi realizado um treinamento prévio com os idosos a fim de minimizar o viés de aprendizado dos testes. O avaliador não estava cego quanto ao grupo ao qual cada idoso pertencia.

Para análise dos dados foi utilizado o programa IBM SPSS versão 20. Foi aplicada estatística descritiva para caracterização da amostra (média, desvio padrão e intervalo de confiança de 95\%). O Teste de ShapiroWilk foi usado para verificar a normalidade dos dados do estudo, o teste $\mathrm{T}$ independente para comparar a idade entre os grupos, o Qui Quadrado para verificar possíveis associações entre variáveis de interesse e participação no Centro de Convivência, e o teste U de Mann-Whitney para comparar os resultados obtidos na Escala de Equilíbrio de BERG, TUG, TAF e domínios do SF-36, entre os grupos GP e GNP.

\section{RESULTADOS}

Participaram do estudo 56 idosos de ambos os gêneros, sendo 28 idosos participantes do Centro de Convivência da Melhor Idade (GP), e 28 idosos moradores da comunidade, não participantes de centros de convivência (GNP).

Na Tabela 1 são apresentadas as características dos participantes quanto à idade, gênero, atividade física, histórico de quedas, doenças associadas e principais medicamentos utilizados. Os grupos foram homogêneos quanto à idade, gênero, histórico de quedas, doenças associadas e principais medicamentos utilizados. Em ambos os grupos houve predomínio do gênero feminino. 
Tabela 1. Variáveis demográficas, atividade física e história clínica de 56 indivíduos idosos, sendo 28 participantes do Centro de Convivência da Melhor Idade (GP), e 28 idosos moradores da comunidade, não participantes de nenhum centro de convivência (GNP). Santo Amaro da Imperatriz, SC, julho/agosto de 2016.

\begin{tabular}{|c|c|c|c|}
\hline Variáveis & $\begin{array}{c}\text { GP }(n=28) \\
n(\%)\end{array}$ & $\begin{array}{c}\text { GNP }(n=28) \\
n(\%)\end{array}$ & $\mathbf{p}^{*}$ \\
\hline Idade (anos) (Média ニDP) & $72,6 \pm 6,9$ & $70,8 \pm 6,5$ & 0,24 \\
\hline Gênero feminino & $21(75 \%)$ & $20(71,4 \%)$ & 0,76 \\
\hline Praticante de atividade física & $28(100 \%)$ & $12(42,8 \%)$ & 0,001 \\
\hline Histórico de quedas & $12(42,8 \%)$ & $15(53,6 \%)$ & 0,42 \\
\hline $\begin{array}{l}\text { Doenças associadas } \\
\text { Hipertensão arterial sistêmica } \\
\text { Doença ortopédica } \\
\text { Hipercolesterolemia } \\
\text { Cardiopatia }\end{array}$ & $\begin{array}{c}14(50 \%) \\
12(42,8 \%) \\
5(17,8 \%) \\
4(14,3 \%)\end{array}$ & $\begin{array}{l}15(53,6 \%) \\
20(71,4 \%) \\
6(21,4 \%) \\
6(21,4 \%)\end{array}$ & 0,19 \\
\hline $\begin{array}{l}\text { Principais medicamentos } \\
\text { Hipocolesterolemiante } \\
\text { Anti-hipertensivo } \\
\text { Tiazídico } \\
\text { Antiulceroso } \\
\text { Hipoglicemiante }\end{array}$ & $\begin{array}{c}9(32,1 \%) \\
15(49,9 \%) \\
8(28,6 \%) \\
8(28,6 \%) \\
4(14,3 \%)\end{array}$ & $\begin{array}{c}8(28,6 \%) \\
11(39,3 \%) \\
3(10,7 \%) \\
1(3,6 \%) \\
7(25 \%)\end{array}$ & 0,71 \\
\hline
\end{tabular}

* Teste T Independente ou Qui Quadrado.

Valores em número amostral e porcentagem, exceto idade (média \pm desvio padrão).

GP, Grupo participante do Centro de Convivência; GNP, Grupo Não Participante do Centro de Convivência.
Houve associação significativa entre a prática de atividade física e a participação no Centro de Convivência $(p=0,001)$, tendo em vista que todos sujeitos do GP praticavam atividade física, ao contrário do GNP, no qual somente $42,8 \%$ faziam algum tipo de atividade física.

A Tabela 2 apresenta os resultados referentes à EEB, TUG e TAF dos dois grupos, sendo observada diferença significativa nos resultados da EEB entre o GP e o GNP $(p=0,001)$, tendo o GP apresentado melhor desempenho. Também foi observada diferença significativa entre os dois grupos avaliados no TUG ( $p=0,001)$, com o GP levando menos tempo para realizar o teste em comparação ao GNP. Já em relação ao TAF não houve diferença entre os grupos $(\mathrm{p}=0,33)$.

$\mathrm{Na}$ Tabela 3 são apresentados os resultados relativos ao questionário de QV SF-36, sendo observada diferença significativa em todos os domínios da avaliação entre os grupos, com maior pontuação e consequente melhor QV no GP.

Tabela 2. Escores obtidos na Escala de Equilíbrio de Berg, no Teste Timed Up and Go e no Teste de Alcance Funcional Anterior pelos participantes do Grupo do Centro de Convivência da Melhor Idade (GP) e dos idosos moradores da comunidade (GNP). Santo Amaro da Imperatriz, SC, julho/agosto de 2016.

\begin{tabular}{|c|c|c|c|c|c|}
\hline & \multicolumn{2}{|c|}{ GP $(n=28)$} & \multicolumn{2}{|c|}{ GNP $(n=28)$} & \multirow{2}{*}{$\mathbf{p}^{*}$} \\
\hline & $\mathrm{M} \pm \mathrm{DP}$ & IC $95 \%$ & $M \pm D P$ & IC $95 \%$ & \\
\hline EEB (pontos) & $53,2 \pm 2,1$ & $52,3-54,1$ & $48,8 \pm 6,2$ & $46,4-51,4$ & 0,001 \\
\hline TUG (segundos) & $9,5 \pm 1,5$ & $9-10,2$ & $13,1 \pm 5,1$ & $11,1-15,2$ & 0,001 \\
\hline TAF $(\mathrm{cm})$ & $20,9 \pm 6,5$ & $18,3-23,6$ & $22,2 \pm 5,5$ & $20,3-24,5$ & 0,33 \\
\hline
\end{tabular}

* Teste U de Mann-Whitney.

GP, Grupo Participante do Centro de Convivência; GNP, Grupo Não Participante do Centro de Convivência; M, média; DP, desvio padrão; IC, Intervalo de confiança de 95\%; EEB, Escala de Equilíbrio de Berg; TUG, Teste Timed Up and Go; TAF, Teste de Alcance Funcional.

Tabela 3. Escores obtidos em cada domínio do Questionário de Qualidade de Vida SF-36 pelos participantes do Grupo do Centro de Convivência da Melhor Idade (GP) e dos idosos moradores da comunidade (GNP). Santo Amaro da Imperatriz, SC, julho/agosto de 2016.

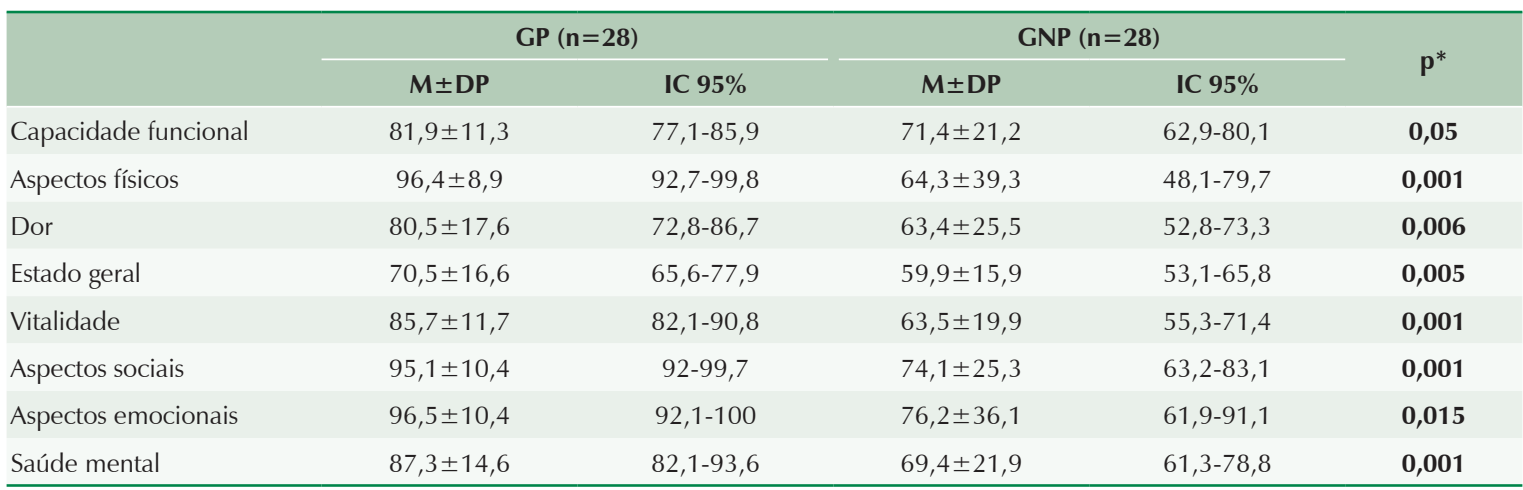

* Teste $U$ de Mann Whitney.

M, média; DP, desvio padrão; IC, Intervalo de confiança de 95\%. GP, Grupo participante do Centro de Convivência; GNP, Grupo não participante do Centro de Convivência. 


\section{DISCUSSÃO}

Considerando o aumento gradativo da população idosa e as perdas que ocorrem nos sistemas responsáveis pela manutenção do controle postural e do equilíbrio com o avanço da idade, justifica-se a relevância do presente estudo, que observou melhor equilíbrio e mobilidade funcional nos idosos participantes de um centro de convivência em relação aos não participantes. Acredita-se que estes achados possam estar associados ao fato de que todos idosos participantes do centro praticavam atividade física, sendo esta uma das principais atividades proporcionadas pelo centro de convivência.

Os achados deste estudo vão ao encontro de Tomicki et al. [17], que observaram melhora no equilíbrio de idosos após participação em um programa de atividades físicas e também concordam com outros estudos [5-8, 18-23], nos quais foram avaliados o equilíbrio e a mobilidade funcional de idosos ativos fisicamente e sedentários, observando menor mobilidade funcional e maiores déficits no equilíbrio nos idosos sedentários, concluindo então que a prática de atividades físicas contribuiu para a redução do risco de quedas.

Adicionalmente, Silva et al. [24], estudando idosas da comunidade, compararam o equilíbrio, a mobilidade e o desempenho muscular segundo a autoeficácia para quedas, ou seja, o grau de confiança que a pessoa tem em realizar atividades do dia a dia sem cair, sendo que as idosas com baixa autoeficácia para quedas apresentaram pior equilíbrio e mobilidade funcional. Esse achado demonstra que o equilíbrio e a mobilidade funcional preservados geram maior autonomia no idoso e menor risco de quedas. No presente estudo não se observou diferença significativa no alcance funcional anterior, avaliado pelo TAF, sendo que os idosos de ambos os grupos apresentaram valores que não indicam risco de quedas.

Segundo Wichman et al. [25], um dos motivos que levam os idosos a frequentar grupos de convivência é a busca pelo aumento nos seus níveis de atividade física, para assim prevenir as perdas funcionais decorrentes do envelhecimento e recuperar suas capacidades físicas, melhorando consequentemente sua QV. Um envelhecimento saudável e socialmente engajado pode representar um bom preditor para uma velhice bem sucedida. $\mathrm{O}$ envelhecimento saudável não é caracterizado somente por uma boa saúde, mas também pela existência de outros fatores que influenciam para que os idosos se sintam ativos. Os centros de convivência podem auxiliar nessa rede de apoio para a busca da autonomia, melhora da autoestima e da resiliência e redução na vulnerabilidade [25].
No presente estudo, observou-se melhor QV em todos os domínios do SF-36 nos idosos que participam do centro de convivência, o que leva a crer que o convívio, a interação social, bem como a prática de exercícios físicos regulares, foram importantes para esse resultado. Estes dados vão ao encontro de outros estudos [11, 26, 27], os quais verificaram que a participação em grupos de convivência amplia o convívio social, além de auxiliar na melhora das condições de saúde bem como no equilíbrio emocional dos participantes, melhorando consequentemente a QV dos mesmos, refletindo desta forma a importância de se viver em grupo.

Muitos idosos buscam a participação em centros de convivência para melhora física e mental através de exercícios físicos. Porém, a participação nestes grupos proporciona também a maior participação em atividades de lazer, como viagens e atividades ocupacionais e lúdicas [25]. Nesse sentido, os centros de convivência proporcionam maior interação, inclusão social e uma maneira de resgatar a autonomia e o viver saudável [25]. Acredita-se que a inserção dos idosos em centros de convivência é de extrema importância na sua $\mathrm{QV}$, tanto no âmbito físico quanto mental [11, 28-30].

Wichman et al. [25], em entrevista com idosos participantes de centros de convivência, relatam que os idosos referem que nesses locais compartilham vários sentimentos, desde angústia e tristeza até alegrias e afetos. Nos centros, eles têm a possibilidade de conversar com amigos e trocar experiências de vida, associando tudo isso a um melhor bem-estar físico e mental. Sendo assim, segundo esses autores, as redes de relações são fontes importantes de suporte social e estão relacionadas ao senso de bem-estar e consequente melhora na QV [25].

Foram consideradas limitações deste estudo o pequeno número de participantes e o fato dos dados terem sido coletados em apenas um centro de convivência, o que dificulta a generalização dos resultados, além do não cegamento do avaliador quanto ao grupo a que cada idoso pertencia no momento da coleta dos dados. Sendo assim, sugerem-se futuros estudos que avaliem um maior número de idosos, que envolvam mais centros de convivência e com avaliadores cegados, para evitar possíveis vieses de aferição e aumentar o poder estatístico da análise.

Embora considerando essas limitações, é possível concluir, pelos resultados deste estudo, que os idosos que participavam de centros de convivência apresentaram melhor equilíbrio, mobilidade funcional e QV quando comparados com idosos da comunidade que não participavam de centros de convivência. 


\section{NOTAS}

\section{Apoio financeiro}

Este estudo não recebeu apoio financeiro de fontes externas.

\section{Declaração de conflito de interesses}

Os autores declaram não haver conflitos de interesses relevantes ao conteúdo deste estudo, informam ter tido acesso a todos os dados obtidos e assumem completa responsabilidade pela integridade dos resultados.

\section{REFERÊNCIAS}

1. Dias DSG, Carvalho CS, Araújo CV. Comparação da percepção subjetiva de qualidade de vida e bem-estar de idosos que vivem sozinhos, com a família e institucionalizados. Rev Bras Geriatr Gerontol. 2013;16(1):127-38. https://doi. org/10.1590/S1809-98232013000100013

2. Oliveira DV, Faria TG, Morales RC, Benedeti MR. Análise da capacidade funcional de idosos institucionalizados à partir da auto-avaliação. Conexões. 2009;7(2):79-95.

3. Woellner SS, Araujo AGS, Martins JS. Protocolos de equilíbrio e quedas em idosos. Rev Neuroc. 2014;10(2):104-17.

4. Meneses SRF; Burke TN, Marques AP. Equilíbrio, controle postural e força muscular em idosas osteoporóticas com e sem quedas. Fisioter Pesqui. 2012;19(1):26-31. https://doi.org/10.1590/S1809-29502012000100006

5. Fernandes AMBL, Ferreira JJA, Stolt LROG, Brito GEG, Clementino ACCR, Sousa NM. Efeitos da prática de exercício físico sobre o desempenho da marcha e da mobilidade funcional em idosos. Fisioter Mov. 2012;25(4):821-30. https:// doi.org/10.1590/S0103-51502012000400015

6. Morris JN, Howard EP, Steel K, Berg K, Tchalla A, Munankarmi A, David D. Strategies to reduce the risk of falling: Cohort study analysis with 1-year follow-up in community dwelling older adults. BMC Geriatr. 2016;16:92. https://doi. org/10.1186/s12877-016-0267-5

7. Hafström A, Malmström EM, Terdèn J, Fransson PA, Magnusson M. Improved Balance Confidence and Stability for Elderly After 6 Weeks of a Multimodal Self-Administered Balance-Enhancing Exercise Program A Randomized Single Arm Crossover Study. Gerontol Geriatr Med. 2016;2:2333721416644149. https://doi.org/10.1177/2333721416644149

8. Gouveia BR, Jardim HG, Martins MM, Gouveia ÉR, Freitas DL, Maia JA, Rose DJ. An evaluation of a nurse-led rehabilitation programme (the ProBalance Programme) to improve balance and reduce fall risk of community-dwelling older people: A randomised controlled trial. Int J Nurs Stud. 2016;56:1-8. https://doi.org/10.1016/j.ijnurstu.2015.12.004

9. Freitas MC, Queiroz TA, Sousa JAV The meaning of old age and the aging experience of in the elderly. Rev Esc Enferm USP. 2010;44(2):403-8.

10. Bueno DR, Marucci MDFN, Roediger MDA, Gomes IC, Duarte YADO, Lebrão ML. Nível de atividade física, por acelerometria, em idosos do município de São Paulo: estudo sabe. Rev Bras Med Esporte. 2016;22(2):108-12. https:// doi.org/10.1590/1517-869220162202148501

11. Both JE, Leite MT, Hildebrandt LM, Pilati ACL, Stamm B, Jantsch LB. Grupos de convivência: uma estratégia de inserção do idoso na sociedade. Contexto \& Saúde. 2013;11(20):995-8.

12. Berg KO, Wood-Dauphinee SL, Williams JI, Maki BE. Measuring balance in the elderly: validation of an instrument. Can J Public Health. 1992;83(2):7-11.

13. Podsiadlo D, Richardson S. The Timed "Up \& Go": a test of basic functional mobility for frail elderly persons. J Am Geriatr Soc. 1991;39(2):142-8. https://doi.org/10.1111/j.1532-5415.1991.tb01616.x

14. Duncan PW, Weiner DK, Chandler J, Studenski S. Functional reach: a new clinical measure of balance. J Gerontol. 1990;45(6):192-7. https://doi.org/10.1093/geronj/45.6.M192

15. Ciconelli RM, Ferraz MB, Santos W, Meinão I, Quaresma MR. Tradução para língua portuguesa e validação e do questionário genérico de avaliação de qualidade de vida SF-36. Rev Bras Reumatol, 1999;39(3):143-50.

16. Ware JE, Snow KK, Kosinnski M, Gandek B. SF-36 Health Survey. Manual and Interpretation Guide. Boston: New England Medical Center; 1993.

17. Tomicki C, Zanini SCC, Cecchin L, Benedetti TRB, Portella MR, Leguisamo CP. Efeito de um programa de exercícios físicos no equilíbrio e risco de quedas em idosos institucionalizados: ensaio clínico randomizado. Rev Bras Geriatr Gerontol. 2016;19(3):473-82. https://doi.org/10.1590/1809-98232016019.150138

18. Padoin PG, Gonçalves MP, Comaru T, Silva AMV. Análise comparativa entre idosos praticantes de exercício físico e sedentários quanto ao risco de quedas. Mundo Saúde. 2010;34(2):158-64. https://doi.org/10.15343/0104-7809.20102158164

19. Nunes NM, Hauser E, Griebler EM, Martins VF, Possamai VD, Gonçalves AK. Avaliação do medo de cair e equilíbrio de idosos ativos e não ativos: um estudo comparativo. RBCM. 2016;24(2):173-81. https://doi.org/10.18511/0103-1716/ rbcm.v24n2p173-181

20. Cardoso FP, Gonçalves AK. Associação entre domínio de capacidade funcional (SF-36), medo de cair e histórico de quedas em idosos ativos. Ciência em Movimento. 2016;18(36):1-8. https://doi.org/10.15602/1983-9480/cm.v18n36p1-8

21. Rodrigues GD, Barbeito AB, Junior EDDA. Prevenção de quedas no idoso: revisão da literatura brasileira. RBPFEX. 2016;10(59):431-7. 
22. Landers MR, Oscar S, Sasaoka J, Vaughn K. Balance confidence and fear of falling avoidance behavior are most predictive of falling in older adults: prospective analysis. Phys Ther. 2016;96(4):433-42. https://doi.org/10.2522/ptj.20150184

23. Wichmann FMA, Areosa SVC, Lepper L, Couto AN, Cardoso CMC, Moreira EP. Satisfação do idoso na convivência em grupos. Contexto \& Saúde. 2013;11(20):491-8. http://dx.doi.org/10.21527/2176-7114.2011.20.491-498

24. Silva CRBP, Guerra RO, Fonsêca AMC, Gomes AC, Maciel ACC. Mobility, balance and muscle performance according to self-efficacy for falls in the elderly. Fisioter Mov. 2015;28(2):231-40. https://doi.org/10.1590/0103-5150.028.002.AO03

25. Wichmann FMA, Couto AN, Areosa SVC, Monta-és MCM. Grupos de convivência como suporte ao idoso na melhoria da saúde. Rev Bras Geriatr Gerontol. 2013; 16(4):821-32. https://doi.org/10.1590/S1809-98232013000400016

26. Gutierrez BAO, Auricchio AM, Medina MVJ. Mensuração da qualidade de vida de idosos em centros de convivência. J Health Sci Inst. 2011;29(3):186-90.

27. Nascimento LCG, Patrizzi LJ, Oliveira CCES. Efeito de quatro semanas de treinamento proprioceptivo no equilíbrio postural de idosos. Fisioter Mov. 2012;25(2):325-31. https://doi.org/10.1590/S0103-51502012000200010

28. De Paula CLM, Santos EVL, Maia PCGGS, Gouveia Filho PS, Sousa MNA. Qualidade de vida de idosos participantes de um grupo de convivência no munícipio de São Mamede-PB. REBES. 2016;6(2):1-7. https://doi.org/10.18378/rebes. v6i2.4018

29. Camões M, Fernandes F, Silva B, Rodrigues T, Costa N, Bezerra P. Exercício físico e qualidade de vida em idosos: diferentes contextos sociocomportamentais. Motricidade. 2016;12(1):96-105. https://doi.org/10.6063/motricidade.6301

30. Almeida EA, Madeira GD, Arantes PMM, Alencar MA. Comparação da qualidade de vida entre idosos que participam e idosos que não participam de grupos de convivência na cidade de Itabira-MG. Rev Bras Geriatr Gerontol. 2010;13(3): 435-44. https://doi.org/10.1590/S1809-98232010000300010 